\title{
Bullous Lichen Planus: A Case Report with Review of Literature
}

\section{Kumari $\mathrm{A}^{* 1}$ and Singh $\mathrm{PK}^{2}$}

${ }^{1}$ Department of Oral Medicine and Radiology, Buddha Institute of Dental Sciences and Hospital, Patna, Bihar, India ${ }^{2}$ Department of Periodontics, Buddha Institute of Dental Sciences and Hospital, Patna, Bihar, India

${ }^{*}$ Corresponding author: Kumari A, Senior Lecturer, Department of Oral Medicine and Radiology, Buddha Institute of Dental Sciences and Hospital, Patna, Bihar, India, E-mail: dranjali0112@gmail.com

Citation: Kumari A, Singh PK (2017) Bullous Lichen Planus: A Case Report with Review of Literature. J Case Rep Stud 5(4): 403. doi: 10.15744/2348-9820.5.403

Received Date: June 28, 2017 Accepted Date: August 28, 2017 Published Date: August 30, 2017

\begin{abstract}
Oral lichen planus is a chronic inflammatory mucocutaneous disease which frequently involves the oral mucosa. A 48-year-old male patient with the chief complaint of burning sensation in bilateral buccal mucosa, upper and lower labial mucosa diagnosed as Bullous lichen planus and treated with systemic prednisolone and Benzydamine oral rinse. Patient was followed for 6 months and was almost free of lesions. The case is reported with review of current modalities in the management of oral lichen planus.
\end{abstract}

Keywords: Bullous lichen planus; Mucocutaneous; Burning sensation

\section{Introduction}

Oral lichen planus is a chronic inflammatory mucocutaneous disease which frequently involves the oral mucosa. This disease has most often been reported in middle-aged patients of 30-60 years of age. The disease affects 0.5-2\% of the population [1]. OLP eruptions usually have a distinct clinical morphology and a characteristic distribution, but OLP may also present a confusing array of patterns and form. Andreasen's classical classification consists of reticular, papular, plaque, atrophic, bullous and erosive forms [2]. This reported case of bullous lichen planus needs attention due to its rare occurrence.

\section{Case report}

A 48-year-old Indian male patient reported to the Department of Oral Medicine and Radiology with the chief complaint of burning sensation of oral cavity since 8 months. Burning sensation was insidious in onset, preceded by eruption of fluid filled vesicles that used to burst shortly, continuous, severe intensity aggravated on having spicy and hot food substances and no relieving factors. His medical history revealed being asthmatic on steroid inhaler. Past dental history revealed visiting dentist for same 4 months back but discomfort continued forcing patient to seek help.

No significant family history. He was married since 20 years with three children. Occupational history revealed patient was small scale businessman, often his life was associated with professional stress and tension as reported by the patient. Non-vegetarian by diet. No history of any deleterious habits. He brushed once daily in horizontal manner using brush and paste. General physical examination revealed that he is moderately built and nourished. Extraoral examination revealed nothing significant along with no cutaneous involvement.

On Intra oral examination mixed red and white lesions, irregular in shape measuring $3 \mathrm{x} 4 \mathrm{~cm}$ seen bilaterally in buccal mucosa extending along the line of occlusion extending supero-inferiorly about $2 \mathrm{~cm}$ on either side, and anteroposteriorly from commissure of the mouth up to retromolar area has central area of erythema surrounded surface by white striae (Figure 1). Lesion was also present in upper and lower labial mucosa (Figure 2) with irregular margins and pseudo membrane slough in both buccal mucosa and upper and labial mucosa, rest of the masticatory mucosa (found on the dorsum of the tongue, hard palate) was normal.

On palpation inspectory findings were confirmed as tender, smooth, and nonscrappable lesion.

Gingiva was observed with generalized gingival inflammation, erythematous, bleeding on probing and tender. Generalized pockets and stain and calculus were present. Considering history and clinical features Bullous lichen planus (LP) was considered as provisional diagnosis with the differential diagnosis Pemphigus Vulgaris or Erosive Lichen Planus. 


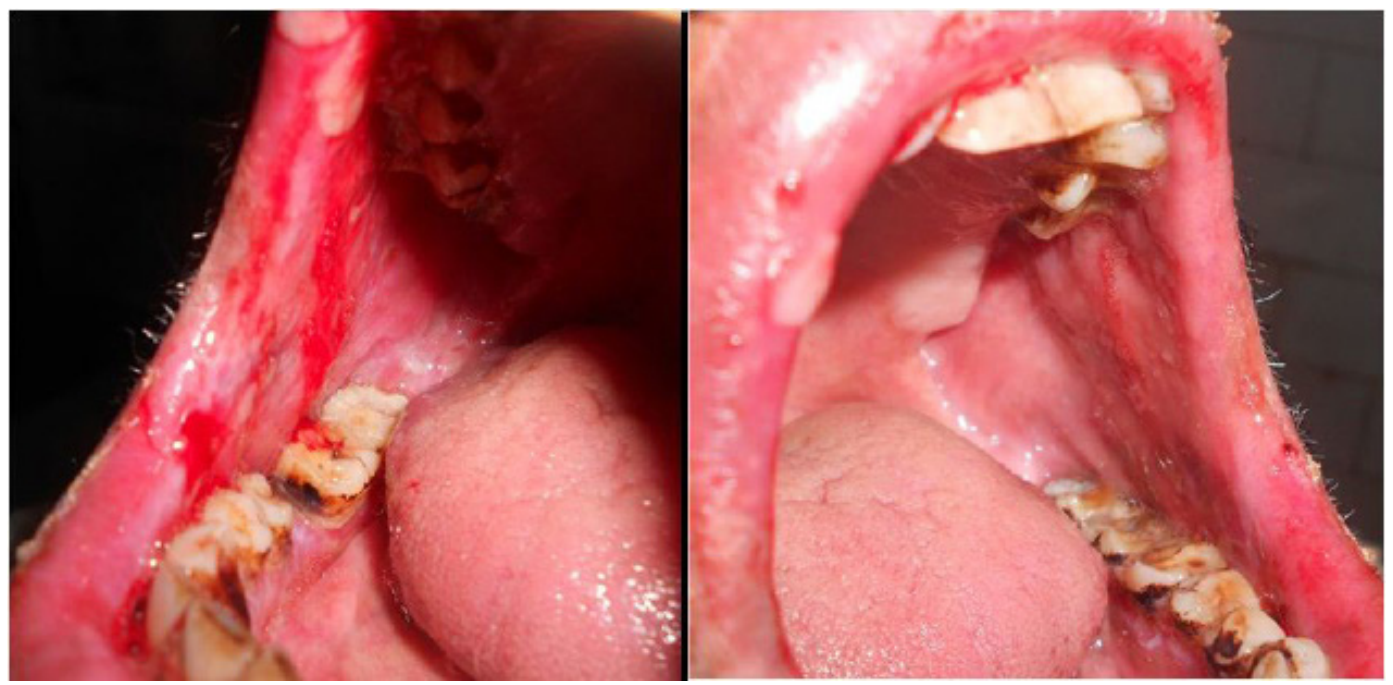

Figure 1: Lesions on right and left buccal mucosa

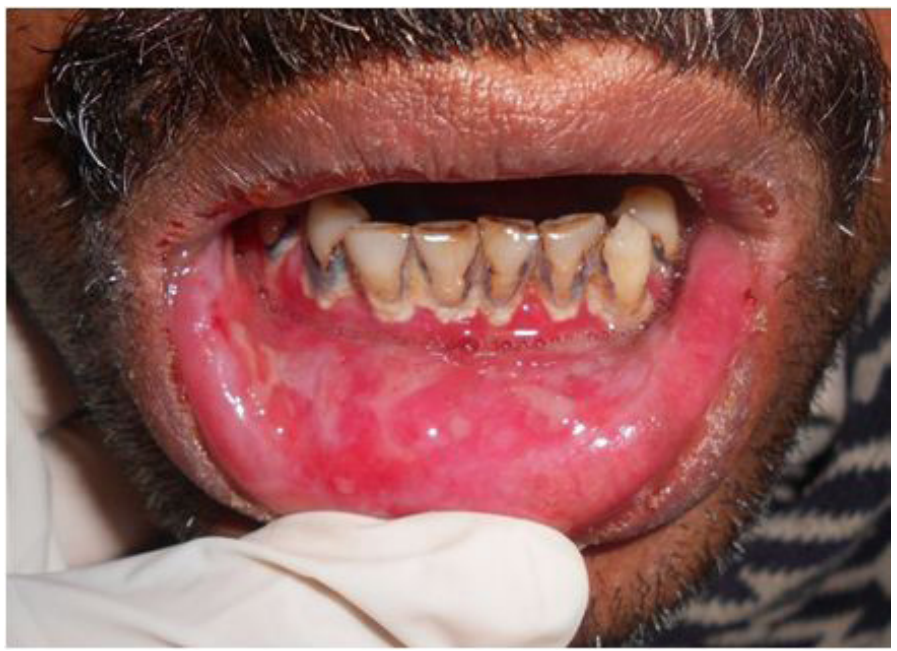

Figure 2: Lesions on lower labial mucosa

Patient underwent investigations like complete hemogram, blood sugar, cytosmear, and incisional biopsy from right buccal mucosa. All laboratory findings were within normal limits, Immunofluorescence assay of specimen using Michel's medium was carried out and result showed negative presence of IgG, IgA and IgM autoantibodies confirming no definite features of Pemphigus (Figure 3) and incisional biopsy (Figure 4) confirmed as bullous LP, which showed typical histopathological features (Figure 5).

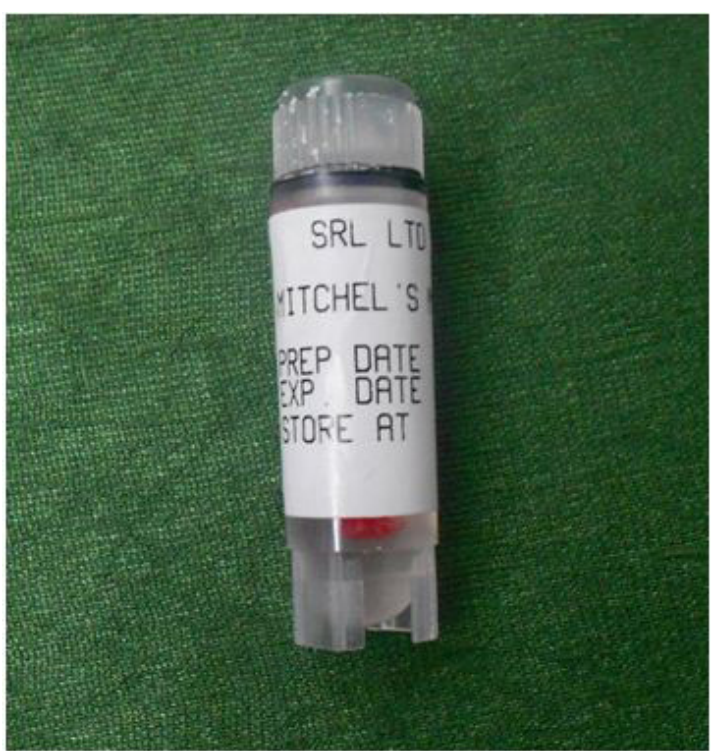

Figure 3: Mitchel's medium for immunofluorescence 


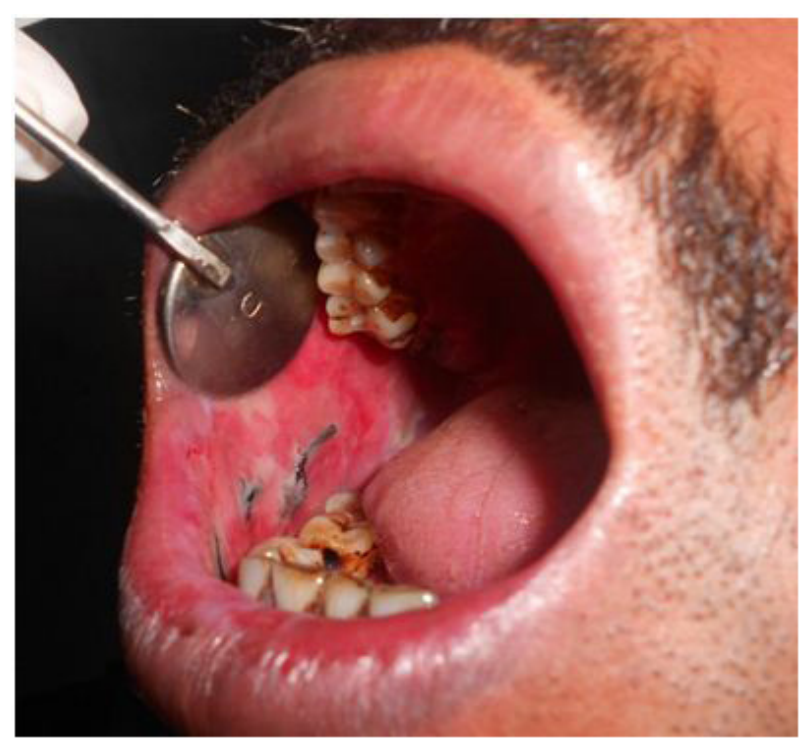

Figure 4: Incisional biopsy from right buccal mucosa

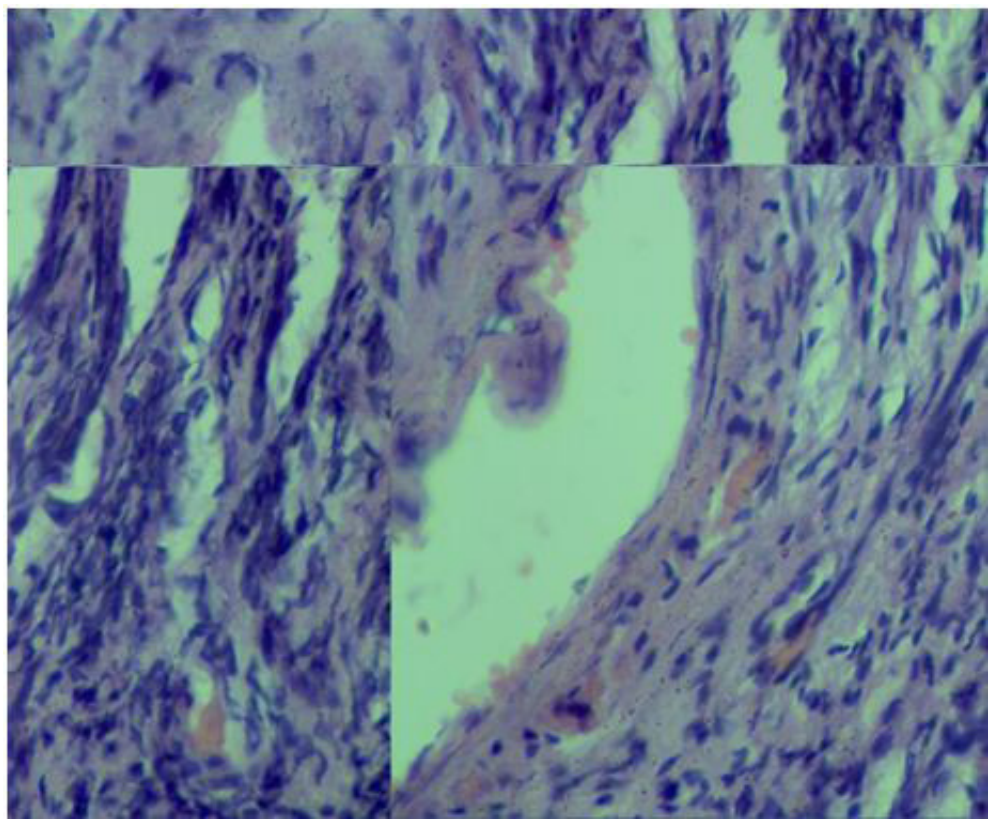

Figure 5: Histopathology showing large Max-Joseph space, saw tooth-like rete pegs, increased granular cell layer

He was treated with Tab prednisolone (Wysolone) $20 \mathrm{mg}$ twice a day for a week followed by $10 \mathrm{mg}$ twice a day for a week, topical clotrimazole (candid 1\%), benzydamine oral rinse $0.15 \%$ (Tantum oral rinse), ranitidine $150 \mathrm{mg}$ (Histac EVT) once daily for 15 days. On second visit after 15 days, patient reported with 50\% reduction in burning sensation. The dose of wysolone was tapered
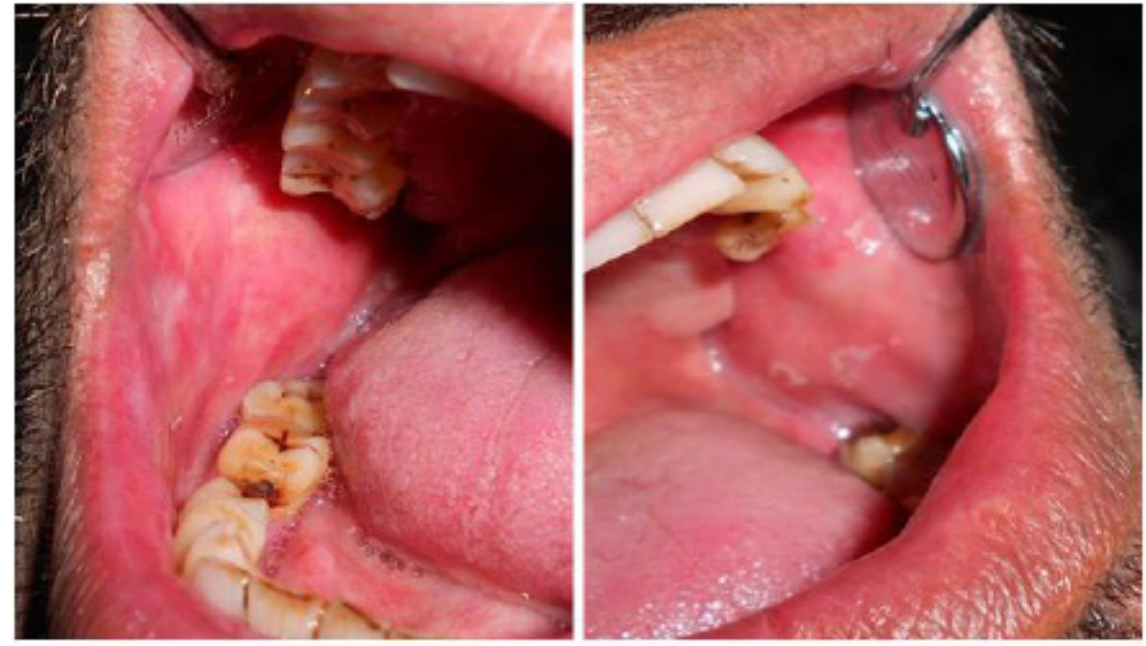


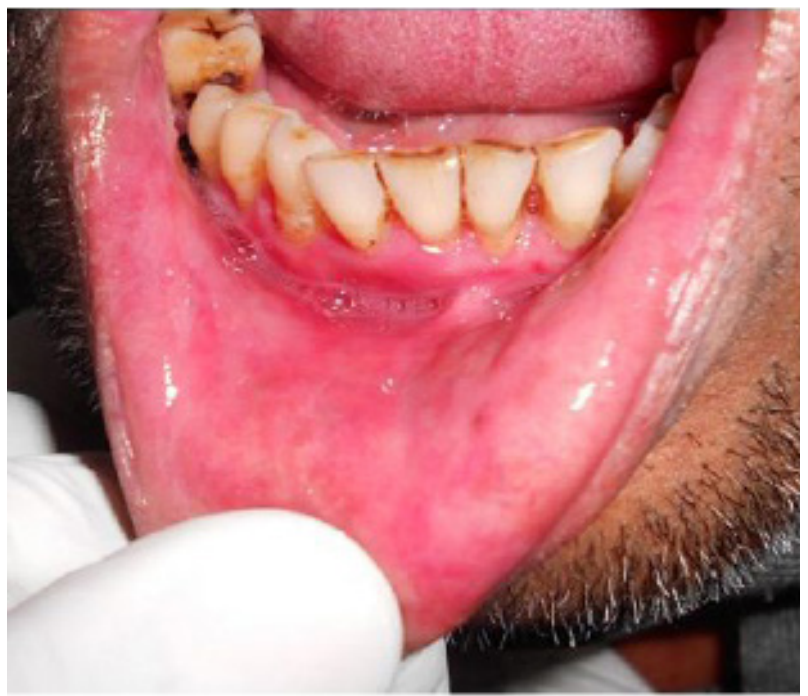

Figure 6: Post treatment-healed lesions of buccal mucosa and labial mucosa

(10 mg once daily), topical clotrimazole was stopped and rest medication continued. On third visit dose of wysolone was again tapered to $5 \mathrm{mg}$ once daily and rest medication was stopped, after 15 days patient reported with complete reduction of burning sensation and was enjoying the taste of food. On fourth visit after 15 days lesions on right and left buccal mucosa had healed for remaining white lesions Triamcinolone (kenocort0.1\%) was prescribed for topical application. On fifth visit his lesions on right and left buccal mucosa and labial mucosa had healed (Figure 6). Patient was referred to Department of Periodontics for oral prophylaxis.

\section{Discussion}

Oral Lichen Planus, (here after known as "OLP”) is chronic inflammatory mucocutaneous disease. Name of condition was provided by British physician, Erasmus Wilson, who first described it in 1869. OLP is reported to occur in 0.5-2\% of population with peak incidence in 30-60 years range [3]. OLP eruptions usually have a distinct clinical morphology and a characteristic distribution, but OLP may also present a confusing array of patterns and form. Andreasen's classical classification consists of reticular, papular, plaque, atrophic, bullous and erosive forms [2]. Bullous form of OLP is quite rare. The intraoral bullae rupture soon after they appear, resulting in the classic appearance of erosive OLP.

Significant pain and discomfort accompany the atrophic, erosive, and bullous forms [4]. OLP has been associated with multiple etiological factors such as autoimmunity, anxiety, viral infection, genetic predisposition etc. According to PB Sugarman OLP is a T cell-mediated autoimmune disease in which auto-cytotoxic CD8 T cells trigger apoptosis of oral epithelial cells as basal epithelial cells are recognized as foreign because of changes in the antigenicity of their cell surface $[5,6]$.

A complete history and physical examination are usually sufficient to make a clinical diagnosis of OLP. However, biopsy is required to differentiate between OLP and other chronic white or ulcerative oral lesions including reactive keratoses, chronic hyperplastic candidosis and epithelial dysplasia. Malignancy must also be excluded. Direct immunofluorescence can help distinguish erosive, ulcerative or the very rare bullous form of OLP from pemphigus vulgaris, benign mucous membrane pemphigoid, dermatitis herpetiformis and linear IgA, bullous dermatosis [7].

\section{Management}

Lichen planus characterized by relapse and remissions, so its management should aim at the resolution of painful symptoms, oral mucosal lesions, the risk of oral cancer and the maintenance of good oral hygiene. No treatment modality has been proved curative for OLP; switching on to the alternative agents used in the management of OLP suggests the inadequacy of any one agent to provide relief to the patient. Various treatment modalities for lichen planus have been tried including topical and systemic steroids, retinoids, immunosuppressive drugs, surgery, lasers and Photochemotherapy. Presently, treatment of lichen planus aimed at alleviating symptoms during periods of exacerbation and to prolong the duration of remission. Currently, treatment for OLP is focused mainly to eliminate mucosal erythema, ulcerations and alleviate symptoms disease during periods of activity and, if possible, increase the periods of disease quiescence.

To date, corticosteroids remain the first choice of treatment for OLP, it has been found to be the most expected and successful agents in the treatment of OLP. Currently, corticosteroids are the drug of choice for the treatment of OLP. It may be employed in the form of topical superficial application or intralesional injection; or systemic or combination of topical and systemic. Topical aqueous triamcinolone acetonide suspension is proven to be effective in reducing mucosal erythema and ulcerations [7]. Topical tacrolimus $0.1 \%$ in Oraguard-B was effective and safe in treating patients with OLP [8]. Intralesional injection of corticosteroid for recalcitrant or extensive lesions [9]. Systemic steroid therapy should be reserved for patients in whom OLP lesions are recalcitrant 
to topical steroid management [4].

\section{Conclusion}

OLP is a chronic disease with diverse clinical manifestations. The management of OLP should begin by taking the proper history and clinical examination. Incisional biopsy should be done to confirm the diagnosis. Long-term follow-up is needed to assess the malignant potential of the lesion.

\section{References}

1. Boorghani M, Gholizadeh N, Taghavi Zenouz A, Vatankhah M, Mehdipour M (2010) Oral Lichen Planus: Clinical Features, Etiology, Treatment and Management; A Review of Literature. J Dent Res Dent Clin Dent Prospect 4: 3-9.

2. Seoane J, Romero MA, Varela-Centelles P, Diz-Dios P, Garcia-Pola MJ (2004) Oral lichen planus: a clinical and morphometric study of oral lesions in relation to clinical presentation. Braz Dent J 15: 9-12.

3. Shah P, Shah M, Parikh K, Khan F (2010) Oral lichen planus: Etiology,diagnosis and treatment: A review of literature. J of Den Sci 3: 37-40.

4. Edwards PC, Kelsch R (2002) Oral lichen planus: clinical presentation and management. J Can Dent Assoc 68: 494-9.

5. Rhodus NL, Myers S, Kaimal S (2003) Diagnosis and management of oral lichen planus. Northwest Dent 82: 17-25.

6. Sugerman PB, Savage NW (2002) Oral lichen planus: causes, diagnosis and management. Aust Dent J 47: 290-7.

7. Gujjar P, Zingade J, Patil S, Hallur J (2015) Recent Update on Treatment Modalities of Oral Lichen Planus - A Review. IJSS Case Reports \& Reviews 2: 40-4.

8. Malik U, Gupta S, Malik SD, Vashishth S, Zaheeruddin, et al. (2012) Treatment of symptomatic oral lichen planus (OLP) with $0.1 \%$ tacrolimus powder in Oraguard-B - A pilot prospective study. Saudi Dent J 24: 143-148.

9. Jayachandran S, KoijamSashikumar S (2012) Management of Oral Lichen Plannus: A Clinical Study. JIMSA 25: 205-8.

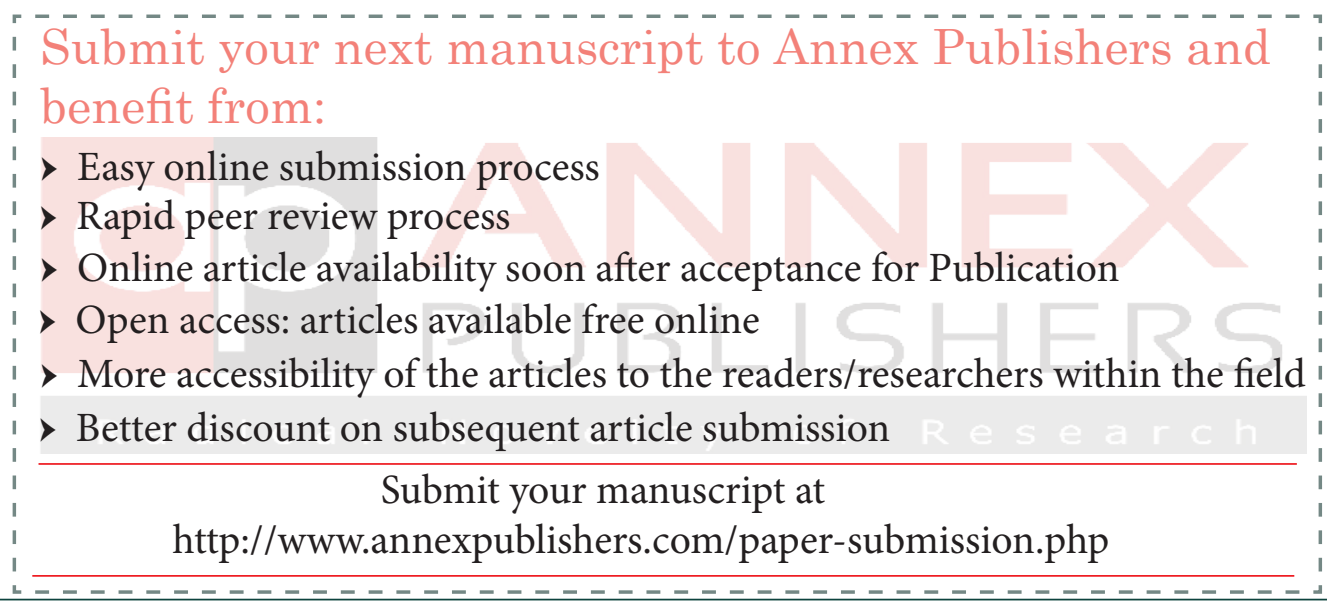

